\title{
Défis éthiques autour des nanotechnologies
}

\section{Jean Martin*}

* Le Dr Martin, privat-docent, est membre du Comité internationa de bioéthique de l'UNESCO et de la Commission nationale suisse d'éthique.
Correspondance:

Dr Jean Martin

La Ruelle 6

CH-1026 Echandens

jean.martin@urbanet.ch
Les développements scientifiques actuels offrent des perspectives dont l'auteur de ces lignes, au terme d'une carrière de santé publique où il s'est beaucoup intéressé à l'éthique, constate qu'elles ont des côtés bousculants, décoiffants - et plus que cela! Au plan conceptuel déjà; quant au plan éthique largement considéré, on est parfois pour ce qui me concerne dans l'inoui, le jamais imaginé. Par exemple dans le domaine des neurosciences (voir un billet récent dans ce même journal [1]), de la procréation et de ses actuels ou possibles avatars [2] ainsi qu'en matière de nanotechnologie. A ce dernier sujet, sont présentés ci-dessous des extraits d'un ouvrage [3] publié par l'UNESCO, l'Organisation des Nations Unies pour l'éducation, la science et la culture.

L'important dans ce livre est non seulement les thèmes abordés, d'ores et déjà discutés dans les cercles scientifiques, mais le fait que sa publication intervient sous les auspices d'une organisation internationale, et particulièrement de la Commission mondiale d'éthique des connaissances scientifiques et des technologies, la COMEST (qui, avec un mandat centré sur les technologies, est une instance-sœur, pourrait-on dire, du Comité international de bioéthique [4]). L'ouvrage n'est actuellement disponible qu'en anglais et la traduction d'éléments substantiels pourra intéresser le lecteur francophone. A noter que le Comité consultatif national français d'éthique pour les sciences de la vie et de la santé (CCNE) a publié cette année un avis sur nanosciences et nanotechnologies [5].

Une caractéristique de l'éthique aujourd'hui est qu'elle n'est pas seulement en rapport avec la science et les développements scientifiques mais comprend une dimension culturelle et pédagogique essentielle, dit l'UNESCO. La priorité que l'organisation lui accorde depuis une quinzaine d'années illustre que l'éthique de la science et de la technologie doit être considérée, de plus en plus, comme une responsabilité générale de la communauté politique mondiale.

\section{Nanotechnologies, Ethics and Politics}

C'est le titre du livre auquel nous faisons référence [3], fondé sur le travail d'un groupe d'experts chargé de présenter un état de la situation. $\mathrm{Au}$ niveau international en effet, il importe de déterminer quelles décisions de politique (policies) sont nécessaires. Il convient de demander notamment si et comment le développement des nanotechnologies peut être orienté en fonction de buts sociaux et environnementaux plus larges.

Dans son introduction, Henk ten Have, directeur de publication, relève que, en pratique quotidienne, il est évident que l'éthique joue seulement un rôle mineur dans l'évaluation des technologies (ET - technology assessment/TA en anglais). Plutôt que d'attendre que les questions éthiques surgissent, il serait bien préférable en vue de la prise d'options de policy qu'une approche prospective de détection avancée des enjeux soit adoptée: «L'évaluation des technologies semble osciller entre deux pôles: une conception étroite qui se concentre sur les questions d'efficacité, sécurité et impact économique des technologies, et une conception large qui prend en compte leurs conséquences sociales et éthiques. Actuellement, c'est la conception étroite qui domine. L'éthique appliquée est souvent considérée comme une variété de technologie qui chercherait à résoudre, ou au moins à soulager (pacify), les conséquences morales de l'emploi des techniques médicales [...]. La bioéthique s'est développée comme une discipline autonome destinée à apporter une aide dans les pratiques de soins, mais elle est aussi devenue une partie de l'ordre technologique. Elle est souvent dominée, dans son raisonnement, par le modèle de l'ingénieur, se basant sur une rationalité technologique s'adressant à une série donnée de problèmes pratiques et appliquant un nombre limité de principes moraux. Dans cette approche, la bioéthique est utilisée pour rendre gérables et contrôlables certaines potentialités» (p. 25-26).

L'éthique ne peut se limiter à ce rôle, elle ne doit pas simplement être instrumentalisée au profit des développements scientifiques sur lesquels elle se penche. Ten Have: «L'éthique peut contribuer à l'évaluation des technologies en allant au-delà du cadre technologique lui-même. Elle centre alors son analyse sur la question de savoir si une technologie en tant que telle se justifie du point de vue de valeurs morales; elle ne considère pas la technologie comme une donnée qui serait indiscutable, mais part d'une perspective critique postulant que les techniques ne sont 
pas neutres du point de vue des valeurs, qu'au contraire elles sont chargées de valeurs: celles d'acquérir des connaissances nouvelles, de chercher à avoir des enfants, de soulager la souffrance, etc. [...] Dans une telle perspective large, on pose un regard critique, par exemple, sur la notion de spropriété du corps〉 qui tend à renforcer la vue dualiste traditionnelle entre corps et esprit. Cette approche demande d'examiner attentivement les prétentions d'amélioration (enhancement) et d'immortalité souvent implicites dans les progrès déconcertants des technologies liées aux cellules-souches [...]. Cette ligne de recherche présume que l'éthique est d'abord et surtout une entreprise philosophique dont le but est de nous comprendre nous-mêmes, ainsi que notre existence - en termes de ce qui est désirable ou pas, admissible ou répréhensible, bon ou mauvais» (p. 27-28).

\section{Les besoins de régulation en nanotechnologie}

Du point de vue de Joachim Schummer, philosophe de l'Université technique de Darmstadt, «le souci éthique le plus pressant actuellement, s'agissant de nanotechnologie, est l'incapacité d'établir de nouveaux standards toxicologiques en vue de réduire les risques pour la santé et l'environnement. Il importe que les Gouvernements suivent le principe de précaution et promulguent des réglementations avant qu'interviennent la production industrielle et le marketing de produits basés sur des nanoparticules [...]. Au fur et à mesure que des dispositifs (devices) deviennent plus petits, plus intelligents et sont intégrés dans des systèmes, ils seront de plus en plus capables d'effectuer des tâches de manière autonome, sans être détectables ou prévisibles. Cela ébranle les systèmes moraux et légaux basés sur la prémisse que seuls les êtres humains prennent des décisions autonomes, dont ils peuvent être tenus pour responsables. Pour éviter l'érosion du concept de responsabilité et pour qu'un contrôle par l'homme soit maintenu, des régulations sont nécessaires qui, primo, définissent clairement le niveau de contrôle humain nécessaire et la latitude laissée aux dispositifs techniques et, secundo, précisent les responsabilités des fabricants comme des utilisateurs. A noter que plus un device est puissant, plus son usage criminel sera dangereux; en conséquence, dans l'élaboration de règles/lois, il est nécessaire de fixer si et comment la disponibilité de tels produits pour le public doit être limitée» (p. 93-94).

A propos de ce dernier point (accès par le public), on est au centre d'une problématique majeure: les restrictions à la liberté d'agir comme bon lui semble de l'individu - de chaque citoyen, il y a là une dimension civique - ont quelque chose d'un tabou, particulièrement dans un pays ayant la culture politique de la Suisse. Pourtant, aussi peu populaire que ce soit de le dire: comme j'estime pour ma part que des bornes devront être mises à une liberté complète des chercheurs, des limites devront être posées quant à l'emploi tous-azimuts, par tout un chacun, des possibilités ouvertes par les technologies. La mise en œuvre pratique de telles interdictions posera des défis, c'est certain; il faudra les affronter.

\section{Une recherche qui se centre sur les besoins sociétaux}

Schummer encore: «Le présent battage médiatique autour de tout ce qui est nano- (nano-hype) a créé une situation dans laquelle tout ce qui porte cette étiquette est vu comme important et la recherche y relative est aisément financée. Cependant, pour des raisons éthiques, il est souhaitable que l'argent public soit utilisé pour des études en rapport avec des besoins particuliers de la société. Par exemple, on doit discuter s'il est nécessaire de faire tellement de recherche militaire plutôt que portant sur des besoins civils, ou si le enhancement, le perfectionnement de l'être humain, est plus important que le traitement de maladies qui sont des fléaux» (p. 95). Cette réflexion rejoint nos propres préoccupations sur le poids à donner dans l'allocation des ressources, en matière de recherche aussi, à la pertinence sociale de ce qui est envisagé [6]. Nous réalisons que les scientifiques craignent de voir leurs travaux indûment canalisés par des objectifs sociaux, cas échéant politiques ... c'est compréhensible. Toutefois, dans la mesure où les pouvoirs publics investissent des ressources majeures dans la recherche, celle-ci doit prendre en compte les priorités de la collectivité. Dans notre domaine professionnel, on peut rappeler ce fait curieux, certainement discutable, que parmi les grands partenaires au sein du système de santé, l'industrie pharmaceutique est le seul dont les décisions sont prises en fonction d'impératifs commerciaux uniquement. En effet, dans les pays comme le nôtre, elle est le seul de ces grands partenaires dont les organes dirigeants ne comprennent aucune représentation de l'intérêt commun, de l'intérêt général.

Comme cela a été relevé plus haut, la recherche n'est pas neutre du point de vue des valeurs; elle n'est pas insensible à ce qui attire l'attention et l'argent et à des effets de mode: «Le battage médiatique est l'ennemi d'une évaluation et d'une gouvernance de la technologie réfléchies, sereines [...]. Un danger supplémentaire est que 
la nanotechnologie devienne un symbole de modernisme, dans les pays en développement par exemple, et que l'évaluation des technologies se transforme en débat entre modernisme et traditionalisme.» En réalité, la recherche ne peut plus s'imaginer séparée, à distance, du registre politique au sens large: «Dans la mesure où les technologies façonnent de plus en plus notre société et déterminent la manière dont nous vivons, tout le processus de gouvernance des technologies devient une partie majeure, critique, de l'action politique. Les sociétés démocratiques doivent s'adapter à cette situation [...]. La démocratisation de la gouvernance est le meilleur moyen d'assurer que les technologies émergentes soient développées en accord avec les standards éthiques d'une société» (p. 96).

\section{Nanomédecine, «cyborgisation" - notre identité intrinsèque et notre sphère privée partiront-elles en fumée?}

«En ce qui concerne les développements en nanomédecine, nous partons de l'idée que, à court terme, l'essentiel des efforts de recherche se concentrera sur les questions de diagnostic, prévention et traitement. A moyen terme, quand de premiers résultats solides auront été obtenus, leur orientation va glisser - lentement mais sûrement - vers les techniques amélioratives (enhancement). A long terme, ces techniques développeront une grande puissance; nos efforts de nous changer nous-mêmes par la nanomédecine pourraient mener à des situations dans lesquelles il ne sera plus approprié du tout de parler d'êtres humains (Bert Gordijn, du département d'éthique du Centre médical de l'Université Radboud, Nimègue, Pays-Bas, à la p. 108). Intéressant, non?

«Sous l'influence de l'ingéniérie tissulaire (tissue engineering), la distinction entre corps «naturel〉 et corps «manipulé> (contenant des parties artificielles) pourrait devenir de plus en plus floue. A un certain point, elle pourra même disparaître. Les développements en chirurgie esthétique pourront ajouter encore à la confusion [...] . La perception subjective que l'on a de son corps est influencée par toutes sortes d'expériences, les sentiments d'estime de soi, les attentes liées à son environnement social et d'autres facteurs socioculturels. Une chirurgie esthétique extrêmement développée pourrait finalement transformer le corps humain en un objet très similaire à un vêtement, qui peut devenir à la mode puis se démoder. Plus avant, des systèmes bioélectroniques permettant d'améliorer nos propriétés sensorielles et motrices normales deviendront de plus en plus communs [...]. L'usage invasif et à large échelle de tels systèmes perfectionnant des fonctions centrales amènerait presque certainement de la confusion quant à savoir quelles parties ou fonctions appartiennent véritablement (genuinely) à notre corps» (si elles sont des pièces d'origine!) (idem, p. 113-114).

Gordijn poursuit: «Depuis quelque temps, à propos d'options médicales nouvelles, on pense de plus en plus fréquemment dans le public qu'un bon fonctionnement de son organisme peut être obtenu par le remplacement régulier des pièces défectueuses [...]. Le développement physique, les fonctions physiques et des caractéristiques spécifiques sont vues de moins en moins comme fixées par la nature, inaltérables, de plus en plus comme contrôlables et modifiables.» Les nanotechnologies permettront progressivement une <yborgisation du corps humain>, en en faisant un composite homme-technique (ou homme-machine). «Par exemple, le contact avec des bases de données ne sera plus fait par les doigts travaillant sur un clavier d'ordinateur mais directement par le cerveau. La communication avec des personnes se trouvant à distance deviendrait alors possible non seulement par des média comme le téléphone ou la télévision mais aussi de manière permanente par des liaisons de cerveau à cerveau» (p. 114-116).

«Différents effets secondaires/collatéraux sont concevables, susceptibles de faciliter l'accès à la sphère privée d'autres êtres humains», dit Gordijn. Sur de tels effets, mentionnons un autre ouvrage récent par un journaliste scientifique français [7]: «Les puces radio-communicantes laissent entrevoir une société dans laquelle, à tout moment, il sera possible de contrôler les plus intimes détails de la vie privée des citoyens.» On est en plein Brave New World d'Aldous Huxley, ou 1984 de George Orwell. En conséquence, un être humain trouvera de plus en plus difficile de dire quels résultats de ses pensées et de ses actions constituent encore une réflexion/réalisation personnelle...

\section{Posthumanité/posthumanisme?}

Jusqu'ici, par manque d'imagination probablement (!), la plupart d'entre nous considéraient que l'être humain possédait en propre certaines caractéristiques intrinsèques immuables - qui font de lui un homme. Cela ne semble plus exact. Les défis y relatifs lancés à la biomédecine et à la bioéthique me laissent - très - perplexe (8). Nous sommes maintenant sur une trajectoire de visées de perfectionnement de l'espèce, décrites sous le nom de trans- ou posthumanisme. Gordijn encore: «A long terme, la nanomédecine pourrait mener à une transformation radicale de l'espèce 
humaine [...]. Bien que ces scénarios restent du domaine spéculatif actuellement, il est cependant difficile de réfuter l'idée que la nanomédecine pourrait un jour élaborer des technologies de enhancement si sophistiquées et actives qu'elles changeraient l'humanité de manière telle qu'on ne pourrait plus parler d'êtres humains au sens conventionnel du terme. Dans ce contexte, il y a lieu de poser deux questions: 1) Quelles sont les altérations dont l'envergure et les caractéristiques changeraient les êtres humains si radicalement que ceux-ci devraient être vus comme posthumains?, et 2) Est-il éthiquement souhaitable pour l'espèce de pouvoir aborder une existence posthumaine?» (p. 121).

En guise de remarque conclusive, une phrase de K. Choi, de la Ehwa Woman's University de Séoul: «La nanotechnologie représente un potentiel de changement positif énorme, spécialement dans le domaine des soins et de la médecine, mais un débat public ouvert est nécessaire en ce qui concerne les bénéfices comme les risques de ces possibilités, de manière à assurer une compréhension adéquate du public. Les implications éthiques, légales et sociales de la nanotechnologie concernent la sphère privée, la sécurité, des questions environnementales et métaphysiques qui toutes demandent analyse et discussion détaillées, et ensuite des régulations soigneuses et rigoureuses» (p. 199-200). C'est le moins qu'on puisse dire.

\section{Références}

1 Taverna E. Neurozentrismus. Schweiz. Ärztezeitung. 2007;88(23):1033.

2 Martin J. Biomédecine et procréation - Quel avenir pour les variantes technicisées de la manière de faire des enfants dont nous avons l'habitude? Rev Méd Suisse. 2005;1:453-8.

3 Ten Have H (ed.). Nanotechnologies, Ethics and Politics. Paris: UNESCO Publishing; 2007.

4 Martin J. Le Comité international de bioéthique (CIB) de l'UNESCO et la Déclaration universelle sur la bioéthique et les droits de l'homme. Bull Méd Suisses. 2007;88(5):190-3.

5 CCNE (France). 96 Avis «Questions éthiques posées par les nanosciences, les nanotechnologies et la santé», 2007 (www.ccne-ethique.fr).

6 Martin J. L'évolution de la médecine et les droits et devoirs des personnes et de la société. Bull Méd Suisses. 2001;82(4):120-32. Publié en allemand sous forme résumée: Die zukünftige Entwicklung der Medizin - eine Frage der gesellschaftlichen Solidarität. In: Stauffacher W, Bircher J (Hrsg.). Zukunft Medizin Schweiz. Basel: EMH; 2002. S. $44-58$.

7 Alberganti M. Sous l'œil des puces - La RFID et la démocratie. Paris: Actes Sud; 2007.

8 Martin J. Quo Vadis, éthique en biomédecine? Rev Méd Suisse. 2007;3 (sous presse). 